\title{
La transición del capitalismo al comunismo
}

\author{
The transition from capitalism to communism
}

\begin{abstract}
Marianela Scocco *
Resumen: La transición del capitalismo al comunismo es un tema que siempre ha generado un amplio debate en todo el mundo, especialmente en los sectores de izquierda. Por lo general, entre los teóricos clásicos del marxismo se dio por sentado que entre ambos modos de producción media una fase intermedia, a la que se ha denominado socialismo. El socialismo sería entonces, un período de transición que exige la abolición de las clases sociales, la supresión de toda la propiedad privada de los medios de producción, la eliminación de la diferencia entre la ciudad y el campo, así como la diferencia entre el trabajo manual e intelectual. En este artículo analizamos los postulados de algunos de los principales teóricos del marxismo que reflexionaron sobre la transición del capitalismo al comunismo, postulando que entre ambos modos de producción existen fases o etapas intermedias que denominaron de diferente manera.
\end{abstract}

Palabras clave: Transición, capitalismo, socialismo, comunismo

\begin{abstract}
The transition from capitalism to communism is a subject that has always generated a broad debate around the world, especially in the left sectors. In general, among the classical theorists of Marxism, it was assumed that between the two modes of production there is an intermediate phase, which has been called socialism. Socialism would then be a period of transition that demands the abolition of social classes, the suppression of all private property of the means of production, the elimination of the difference between the city and the countryside, as well as the difference between work manual and intellectual. In this article we analyze the postulates of some of the main theorists of Marxism who reflected on the transition from capitalism to communism, postulating that between both modes of production there are stages or intermediate stages that they denominated differently.
\end{abstract}

Keywords: Transition, capitalism, socialism, communism

Recibido: 8 enero 2018

Aceptado: 22 febrero 2018

\footnotetext{
* Licenciada y profesora en Historia. Instituto de Investigaciones Socio-históricas Regionales de Rosario Consejo Nacional de Investigaciones Científicas y Técnicas (ISHIR-CONICET) y Universidad Nacional de Rosario (UNR). maria_nob4@hotmail.com.
} 


\section{Introducción}

En 2017 se recordaron 150 años de la primera edición de El Capital, obra maestra de Karl Marx, 100 años de la Revolución Rusa y 50 años de la muerte de Ernesto Che Guevara en Bolivia. Los tres acontecimientos marcaron la historia de la humanidad y fueron determinantes en la acumulación de fuerzas en la lucha contra el capitalismo, por el socialismo científico.

La conmemoración de estos eventos ha ocasionado un sinnúmero de publicaciones, artículos de revista, congresos y seminarios en el trascurso de 2017. "El Capital, la Revolución Rusa y la guerrilla del Che representan en tres distintos momentos históricos, una lucha sin claudicación contra el capitalismo, el reformismo y la conciliación de clases. Marx demostró la verdadera esencia del capitalismo - la explotación del hombre por el hombre, desarrolló la teoría socialista dotándole un carácter científico, frente al idealismo del socialismo utópico. Lenin desarrolló el marxismo en la época del imperialismo, comprobó el papel del proletariado y su partido, desnudando las claudicaciones del menchevismo y la II Internacional. El Che insiste en el carácter socialista de la Revolución, en la esencia crítica y creadora del marxismo-leninismo, en la formación del hombre nuevo". 1

La versión original alemana del primer tomo de El Capital de Marx fue publicada en el año 1867. Sin embargo, no existe una versión del primer tomo sino varias versiones. El propio Marx publicó en vida la segunda edición alemana entre junio de 1872 y agosto de 1873 con algunas correcciones. También en 1872 se publicó la primera traducción rusa y entre 1972 y 1875 Marx preparó la versión francesa, que debido a sus adaptaciones no es precisamente una traducción. Este magnífico pensador falleció el 14 de marzo de 1883 y luego de su muerte fueron publicadas el resto de las traducciones del primer tomo así como las demás ediciones alemanas y el resto de la obra que Friedrich Engels divide en dos tomos más, conocidos como el segundo y tercer tomo de El Capital.

Pero la obra de Marx no se agota en El Capital ni constituye sólo una revolución en el campo del conocimiento científico de la sociedad capitalista. Como lo hizo el propio Marx en su práctica como activo participe de la Asociación Internacional de Trabajadores en los mismos años en que escribía El Capital (y a lo largo de su vida en la construcción del movimiento revolucionario), no se trata sólo de conocer las tendencias de la sociedad capitalista, sino de transformarla de raíz. Parte de cómo debía ser esa transformación fue expresado por Marx en la Crítica del Programa de Gotha, una obra clave escrita en 1875 con motivo del proyecto de programa que se iba a aprobar en el Congreso de Gotha, en el que se fusionaron la lassalleana Asociación General de Trabajadores de Alemania y el marxista Partido Socialdemócrata Obrero de Alemania, para dar nacimiento a un nuevo partido, el Partido Socialista Obrero de Alemania, que en 1891 adoptó el nombre de Partido Socialdemócrata Alemán (SPD). La obra se publicó recién en 1891.

Al mismo tiempo, con los 100 años de la Revolución Rusa, también se cumplieron 100 años de la publicación de El Estado y la Revolución escrito por Lenin entre agosto y

\footnotetext{
${ }^{1}$ Arancibia, Simón: "Y límpiese bien la boca si dice Revolución”. 27/09/2017. Tinta Roja. Disponible en: https://tinta-roja.com/2017/09/27/limpiese-bien-la-boca-dice-revolucion/.
} 
septiembre de 1917. En su momento, el texto había quedado inconcluso. El plan del autor incluía el análisis de las experiencias de las revoluciones de 1905 y febrero de 1917, pero lo interrumpió la propia Revolución de Octubre. ${ }^{2}$

En el marco de estas conmemoraciones, queremos analizar uno de los temas que siempre ha generado un amplio debate en todo el mundo, especialmente en los sectores de izquierda: la transición del capitalismo al comunismo. Por lo general, entre los teóricos clásicos del marxismo se dio por sentado que entre ambos modos de producción media una fase intermedia, a la que se ha denominado socialismo. El socialismo sería entonces, un período de transición que exige la abolición de las clases sociales, la supresión de toda la propiedad privada de los medios de producción, la eliminación de la diferencia entre la ciudad y el campo, así como la diferencia entre el trabajo manual e intelectual. Sin embargo, es necesario estudiar con mayor profundidad el tema antes de sacar conclusiones apresuradas y absolutas.

En este artículo analizamos los postulados de algunos de los principales teóricos del marxismo. El trabajo se limita al propio Marx, Lenin, Mao Zedong y el Ernesto "Che" Guevara, que reflexionaron sobre la transición del capitalismo al comunismo, postulando que entre ambos modos de producción existen fases o etapas intermedias que denominaron de diferente manera. ${ }^{3}$

\section{Marx y la transformación revolucionaria}

En la Crítica del Programa de Gotha, Karl Marx sostenía que: "entre la sociedad capitalista y la sociedad comunista media el período de la transformación revolucionaria de la primera a la segunda. Y a este período corresponde también un período político de transición, cuyo Estado no puede ser otro que la dictadura del proletariado"4. Marx denominaba a este período como la primera fase de la sociedad comunista. En ella persistirían diferentes elementos del capitalismo, esencialmente porque no se trataría de "una sociedad que se ha desarrollado sobre su propia base, sino que acaba de salir precisamente de la sociedad capitalista" ${ }^{5}$ y conservaría todavía el sello de la vieja sociedad en todos sus aspectos.

En esta fase el productor obtiene de la sociedad lo que le ha dado, es decir, su cuota individual de trabajo. De esta forma, la jornada social de trabajo se compone de la suma de horas de trabajo individual. La sociedad le entrega a cada trabajador un bono que expresa la cantidad de horas de trabajo que ha rendido, y con este bono saca de los depósitos sociales

\footnotetext{
${ }^{2}$ Lenin lo escribió en la clandestinidad, oculto en alguna aldea cercana a Petrogrado. A poco de terminado el libro, se produjo el asalto al Palacio de Invierno del 7 de noviembre de 1917. Ver: Comité para el Centenario de la Revolución de Octubre: "Una obra inconclusa", en Lenin El Estado y la Revolución. Cuadernos de octubre. Edición Centenario. 2017.

${ }^{3}$ Otros teóricos del marxismo también abordaron y debatieron la temática del período de transición, como, por ejemplo; León Trotsky, Rosa Luxemburgo, Paul Sweezy, entre otros. Sin embargo, la elección por los pensadores nombrados responde a que fueron ellos quienes, con mayor claridad, a nuestro entender, desarrollaron las teorías de las fases o etapas intermedias en la transición del capitalismo al comunismo.

${ }^{4}$ Marx, Karl: Crítica del Programa de Gotha. Editorial Progreso. Moscú. 1977. Pág. 28.

5 Ídem. Pág. 16.
} 
de medios de consumo la parte equivalente a esa cantidad de trabajo. "La misma cuota de trabajo que ha dado a la sociedad bajo una forma, la recibe de ésta bajo otra forma distinta" . En este sentido, Marx resaltaba que aquí reina el mismo principio que regula el intercambio de mercancías, en tanto intercambio de equivalentes, en lo que se refiere a la distribución de medios individuales de consumo entre los distintos productores. Pero varía la forma y el contenido, porque nadie puede dar otra cosa que no sea su trabajo y porque nada puede pasar a ser propiedad privada del individuo. Por eso, el derecho igual sigue siendo un derecho burgués, porque sigue implícita una limitación burguesa, ya que el derecho de los productores es proporcional al trabajo que han rendido, es decir, la igualdad se mide por el mismo rasero; por el trabajo.

Sin embargo, los trabajadores son física e intelectualmente diferentes y rinden más o menos trabajo o pueden trabajar más o menos tiempo, y el trabajo como medida se determina por la intensidad o duración. De esta forma, este derecho igual es un derecho desigual para los trabajos desiguales. Por otro lado, los trabajadores tienen diferentes necesidades, unos están casados y otros no, unos tienen más hijos que otros, etcétera. Por lo tanto, a igual rendimiento, unos obtienen más que otros. Para evitar estos inconvenientes el derecho tendría que ser desigual, pero, según Marx esto era inevitable en la primera fase de la sociedad comunista y era una característica inherente de ésta.

Esto sólo podrá logarse en la fase superior de la sociedad comunista, cuando haya desaparecido la subordinación de los individuos a la división del trabajo y la oposición entre el trabajo intelectual y el manual, cuando el trabajo no sea solamente un medio de vida sino una necesidad, cuando crezcan las fuerzas productivas. Sólo entonces podrá suprimirse el derecho burgués y postularse la demanda hecha por Marx: “¡De cada cual, según su capacidad; a cada cual, según sus necesidades!"’7.

\section{Lenin y la dictadura del proletariado}

Lenin tomó estas ideas marxistas para tratar de ampliarlas en su libro El Estado y la Revolución, escrito en la clandestinidad entre agosto y septiembre de 1917, antes de que se produzca la Revolución de Octubre en Rusia. El revolucionario planteaba que, en la primera etapa del comunismo, la que reconocía como socialismo, los medios de producción han dejado de ser propiedad privada de los individuos y pertenecen a toda la sociedad. Cada miembro de la sociedad, al realizar un trabajo, obtiene de la sociedad un certificado (en Marx un bono) que le acredita haber realizado una determinada cantidad de trabajo y por este certificado recibe los medios de consumo que le corresponden. Luego, el revolucionario ruso continuaba el análisis marxista en relación al derecho igual para hombres distintos. Entonces, según Lenin, la primera fase del comunismo no podrá proporcionar todavía justicia ni igualdad, “(...) pero no será posible ya la explotación del hombre por el hombre, puesto que no será posible apoderarse, a título de propiedad privada, de los medios de producción" ". De esta forma, la sociedad comunista en sus comienzos, se

\footnotetext{
6 Ídem. Pág. 17.

7 Ídem. Pág. 18.

${ }^{8}$ Lenin: El Estado y la Revolución. Editorial Agora. Buenos Aires. 2003. Pág. 97.
} 
verá obligada a eliminar solamente que los medios de producción sean apropiados por individuos aislados, pero no estará en condiciones de evitar que la distribución de los medios de consumo sea según el trabajo, y no según las necesidades. Esto es un "defecto" pero es un defecto inevitable, pues, "no se puede pensar, que al derrocar al capitalismo, los hombres aprenderán a trabajar inmediatamente para la sociedad sin sujetación a ninguna norma de derecho; además, la abolición del capitalismo no sienta de repente tampoco las premisas económicas para este cambio"".

En el socialismo desparecerá, para Lenin, la diferencia entre trabajo intelectual y manual, que es una de las fuentes más importantes de la desigualdad social. Con ello se dará la base económica para la extinción completa del Estado en la sociedad comunista. Por otro lado, con la expropiación de los capitalistas, que debe darse en esta primera fase, se dará la posibilidad de desarrollar ampliamente las fuerzas productivas. Pero, según Lenin, "lo que no sabemos ni podemos saber es la rapidez con que avanzará este desarrollo", hasta terminar con la división del trabajo, suprimir la diferencia entre trabajo intelectual y manual y convertir el trabajo en la primera necesidad de la vida. Finalmente, el Estado se extinguirá, cuando los hombres estén habituados a respetar las reglas fundamentales de convivencia y cuando su trabajo sea tan productivo, que trabajen voluntariamente según sus capacidades. La distribución de los productos no obligará a la sociedad a regular la cantidad de medios de consumo que cada trabajador reciba, sino que todos podrán tomar libremente lo que cumpla a sus necesidades.

Pero mientras se llega a la fase superior del comunismo, "los socialistas exigen el más riguroso control por parte de la sociedad y por parte del Estado sobre la medida del trabajo y la medida del consumo, pero este control sólo debe comenzar con la expropiación de los capitalistas, con el control de los obreros sobre los capitalistas"

Lenin postulaba entonces que a raíz del análisis de Marx se puede hablar de fases de madurez económica del comunismo. En la primera fase, el comunismo no puede presentar todavía una madurez económica completa, no puede aparecer todavía completamente libre de las huellas del capitalismo. De ahí que subsista no sólo el derecho burgués, sino también el Estado burgués que lo garantiza, ahora sin burguesía. Pero, además, se deben tomar otras premisas económicas del propio capitalismo. Ellas son la instrucción general y la formación y educación de la disciplina de los trabajadores por los amplios y complejos aparatos de los correos, de los ferrocarriles, de las fábricas, etcétera. A través de estas premisas se pasa a derrocar a los capitalistas y a los burócratas, a sustituirlos en el registro del trabajo y de los productos. De esta forma, para el líder de la revolución soviética, registro y control es lo que falta para que funcione bien la primera fase de la sociedad comunista. Entonces, todos los ciudadanos serán empleados a sueldo del Estado.

Cuando la mayoría del pueblo empiece a llevar a cabo ese registro y ese control, toda la sociedad será una sola fábrica y una sola oficina, con trabajo y salario iguales. Pero esta disciplina fabril, no es objetivo del comunismo, sino sólo un escalón necesario para limpiar a la sociedad de la explotación capitalista y para seguir avanzando.

\footnotetext{
9 Ídem. Págs. 98 y 99.

${ }^{10}$ Ídem. Pág. 101.
} 
Cuando todos dirijan por su cuenta la producción social, escapar a ese registro hecho por el pueblo será tan difícil que provocará la necesidad de observar que las reglas de convivencia humana se convertirán en una costumbre. Y entonces, se pasará a la fase superior del comunismo y a la extinción del Estado.

Posteriormente, en 1919, después de la revolución bolchevique, Lenin volvió sobre estos temas en su artículo "La economía y la política de la dictadura del proletariado"11. Allí afirmaba que la economía de la Unión Soviética en la época de la dictadura del proletariado, es decir, en el socialismo, representaba la lucha que en sus primeros pasos sostenía el trabajo mancomunado al modo comunista contra la pequeña producción mercantil, contra el capitalismo que seguía subsistiendo y contra el que revivía sobre la base de esta producción. Lenin hablaba de los "primeros pasos" ya que las condiciones necesarias para alcanzar el comunismo se habían realizado sólo en parte, en su fase inicial.

El trabajo estaba mancomunado en la Unión Soviética a la manera comunista por cuanto, "primero, está abolida la propiedad privada sobre los medios de producción y, segundo, porque el Poder proletario del Estado organiza en escala nacional la gran producción en las tierras y empresas estatales, distribuye la mano de obra entre las diferentes ramas de la economía y entre las empresas, distribuye entre los trabajadores inmensas cantidades de artículos de consumo pertenecientes al Estado"12. En este sentido, si bien se habían hecho grandes avances en algunos temas (fue abolida la propiedad privada de la tierra y fueron expropiados sin indemnización los grandes propietarios de la tierra y casi todos los grandes capitalistas; la organización de la gran producción industrial por el Estado, el tránsito del "control obrero" a la "administración obrera" de las fábricas y ferrocarriles, estaba ya realizado en sus rasgos más importantes) con respecto a la agricultura los cambios recién estaban empezando a surgir. Así, apenas había comenzado la organización de las diferentes formas de cooperación de los pequeños productores, como tránsito de la pequeña producción agrícola mercantil a la agricultura comunista. Lo mismo sucedía con la organización estatal de la distribución de los productos en sustitución del comercio privado (el acopio y el envío de cereales a las ciudades y de los artículos industriales al campo por el Estado). La economía campesina continuaba siendo una pequeña producción mercantil. Aquí podía aparecer la base para la restauración del capitalismo, según Lenin.

Por otro lado, el líder bolchevique introdujo en este artículo la cuestión de la lucha de clases. "Socialismo significa la abolición de las clases"13. Para abolir las clases se deben derribar a los terratenientes y a los capitalistas. Para Lenin esta parte de la tarea estaba cumplida en la Unión Soviética. Pero para abolir las clases, es necesario, en segundo lugar, suprimir la diferencia entre los obreros y los campesinos, convertir a todos en trabajadores. Sin embargo, Lenin reconocía que esta es una tarea mucho más difícil. Sólo se puede resolver mediante la reorganización de toda la economía social, pasando de la pequeña producción mercantil, individual y aislada, a la gran producción colectiva. Para esto, el proletariado, debe trazar una línea fundamental, entre los campesinos trabajadores y los

\footnotetext{
${ }^{11}$ Lenin: "La economía y la política de la dictadura del proletariado". Publicado en Pravda, núm. 250, 7 de noviembre de 1919.

12 Ídem. Pág. 2

${ }^{13}$ Ídem. Pág. 4.
} 
campesinos propietarios. "En esta delimitación reside toda la esencia del socialismo"14. El campesino trabajador ha estado oprimido durante siglos por los terratenientes y los capitalistas, ha ido formando su odio y su rechazo contra sus opresores y explotadores, y esto es lo que obliga a los campesinos a buscar la alianza con los obreros contra el capitalista. Pero, al mismo tiempo, las circunstancias de la economía mercantil, hacen que los campesinos sean también pequeños patronos, propietarios y comerciantes aislados.

De esta forma, Lenin afirmaba que las clases han quedado y quedarán durante el socialismo. Precisamente, éste desaparecerá cuando desaparezcan las clases. Sin embargo, a pesar de su preexistencia, las clases han variado las relaciones entre ellas. La lucha de clases no desaparece bajo la dictadura del proletariado, lo único que hace es adoptar otras formas.

\section{Mao Zedong, la Nueva Democracia y el socialismo}

Por su parte, también encontramos reflexionando sobre estos temas a Mao Zedong. El líder revolucionario, en artículos escritos en 1940, previo a que se desencadene la Revolución China de 1949, planteaba que la revolución china se debía dividir en dos etapas. Primero, la democrática, que él denominaba Nueva Democracia, porque ya no pertenece a la vieja categoría, y segundo, el socialismo.

La revolución democrática, para Mao, había comenzado en la Revolución de 1911 en China, pero cambió de rumbo a partir de la Primera Guerra Mundial y de la Revolución de Octubre de 1917 en Rusia. Antes era una revolución democrático-burguesa, bajo la hegemonía de la burguesía china. A partir de dichos acontecimientos, pasó a ser una revolución destinada a establecer una sociedad de nueva democracia y un Estado de dictadura conjunta de todas las clases revolucionarias, pero esta vez dirigida por el proletariado. Esta revolución combatió al imperialismo y al feudalismo. Luego de esta primera etapa, "será el momento de llevar la revolución a su segunda etapa: el establecimiento en China de una sociedad socialista" 15 .

La Nueva Democracia debe ser nueva no sólo en su política, sino también en su economía. Así, Mao planteaba que los grandes bancos y las grandes empresas industriales y comerciales debían ser propiedad del Estado: "El sector estatal de la economía será de carácter socialista y constituirá la fuerza dirigente de toda la economía nacional" ". Sin embargo, Mao aclaraba que no se confiscará el resto de la propiedad privada capitalista, ni se prohibirá el desarrollo de la producción más avanzada que no pueda dominar el pueblo, porque él mismo reconocía que la economía china se encontraba en aquel momento todavía muy atrasada. Por otro lado, se debían adoptar medidas para confiscar la tierra de los grandes terratenientes para distribuirlas entre los pequeños campesinos o los campesinos sin tierra. Estas medidas, para Mao, eran necesarias para abolir las relaciones feudales que aún persistían en el campo (precisamente por no haber concluido aún la primera etapa de la

\footnotetext{
14 Ídem.

${ }^{15}$ Mao, Zedong: "Sobre la nueva democracia. Enero de 1940”. En Obras Escogidas de Mao Tse-Tung. Tomo II. Editorial del Pueblo. Pekín. 1976. Pág. 301.

16 Ídem. Pág. 368.
} 
revolución) y para convertir la tierra en propiedad privada de los campesinos. Seguidamente, aclaraba: "no se establecerá aún en esta etapa una agricultura socialista; no obstante contendrán elementos de socialismo las diversas formas de economía cooperativa que se desarrollen sobre la base de "La tierra para el que la trabaja"17.

Más adelante, Mao formulaba una crítica al Manual de Economía Política de la Unión Soviética de $1960^{18}$, en donde se trataba también el problema de la transición desde un punto de vista más económico y con la experiencia de la Revolución China en marcha. Los revisionistas soviéticos, autores del Manual, afirmaban que: "El período de transición comienza por el establecimiento del régimen proletario y termina con la conclusión de las tareas revolucionarias socialistas, es decir por el establecimiento del socialismo, primera etapa del comunismo" "19. Lo que hizo Mao es preguntarse si esa transición se da solamente hacia el socialismo o si era necesario teorizar también sobre la transición al comunismo, lo que parece un olvido de la teoría marxista por parte de los revisionistas soviéticos, y planteaba la necesidad de establecer una fase más que medie entre el capitalismo y el socialismo. El socialismo, por su parte, también será eliminado para pasar al comunismo. Sin embargo, el comunismo tampoco es una fase estática, sino que también presentará cambios cualitativos y se será dividido en etapas. ${ }^{20}$

Así, Mao afirmaba que China se encontraba en ese momento en la primera fase del comunismo, ya que, en las comunas populares chinas, si bien ya no existía una propiedad individual de los medios de producción, si existía una propiedad colectiva, que aún no era una propiedad social, sino que continuaba siendo propiedad privada. Se pasará a la siguiente fase cuando se llegue al sistema de la propiedad de todo el pueblo.

Asimismo, reflexionaba sobre la permanencia de esta primera fase afirmando que se necesitan muchos años ("hasta cincuenta años") para destruir definitivamente los vestigios de la burguesía y su influencia. Y así ponía como ejemplo al campo: "La concepción del mundo de los campesinos no podrá ser transformada progresivamente, y después completamente, hasta cuando el sistema de la propiedad colectiva de las comunas populares se haya aplicado, y transformado después en sistema de la propiedad nacional, hasta cuando ciudades nuevas y grandes industrias se hayan creado a lo ancho de todo el país, hasta cuando los medios de transporte de todo el país se hayan modernizado y hasta cuando la situación económica haya sido transformada completa y realmente"21. Mao también afirmaba haber llevado a cabo en China una política de confiscación de los bienes de los capitalistas burocráticos, con el fin de realizar el sistema de la propiedad colectiva. Pero a la vez se preguntaba si era necesario hacer lo mismo con los capitalistas medianos y pequeños.

Por otro lado, en el Manual se afirmaba que la base económica de la vía socialista es desarrollar las grandes industrias para eliminar los vestigios del capitalismo. Por su parte,

\footnotetext{
17 Ídem.

${ }^{18}$ Mao, Zedong: La Construcción Del Socialismo. Editorial La Oveja Negra. Medellín. 1975. Se estima que estos textos de Mao fueron escritos entre 1949 y 1968.

${ }^{19}$ Citado en Mao, Zedong: La Construcción Del Socialismo. Editorial La Oveja Negra. Medellín. 1975. Pág. 17.

${ }^{20}$ Mao, Zedong: Discurso: Sobre problemas de filosofía. 18 de agosto de 1964.

${ }^{21}$ Mao, Zedong: La Construcción Del Socialismo. Págs. 29 y 30.
} 
Mao sostenía que no es necesario tener previamente fuerzas productivas plenamente desarrolladas para poder transformar las relaciones de producción caducas. Ahora bien, "Primero hay que derrocar la antigua superestructura por medio de la revolución para que las antiguas relaciones de producción puedan ser abolidas. Es después de la eliminación de éstas cuando se pueden crear nuevas relaciones de producción, abriendo un camino al desarrollo de las fuerzas productivas de la nueva sociedad."22

En cuanto a los sistemas de propiedad, los revisionistas soviéticos decían, y en esto coincidía Mao, que un país que está construyendo el socialismo, no puede basarse durante un período relativamente largo sobre dos sistemas de propiedad diferentes, es decir, sobre la industria socialista más grande y más unificada, el sistema de la propiedad de todo el pueblo, por un lado, y sobre la base de una economía campesina de pequeña producción mercantil dispersa y atrasada, por el otro. Lo que no decían los revisionistas y que resaltaba Mao, es que la contradicción entre estos dos sistemas es en realidad la contradicción entre los obreros y los campesinos. Además, a medida que se prolonga, esta coexistencia responde cada vez menos a las necesidades del desarrollo de las fuerzas productivas. No se puede responder a las necesidades del aumento constante del nivel de vida de los campesinos y de la producción agrícola, y del incremento continuo de las materias primas necesarias para la industria. "Si se quiere satisfacer esas necesidades, se está obligado a resolver la contradicción entre los dos sistemas de propiedad, a transformar el sistema de propiedad colectiva en sistema de propiedad de todo el pueblo y a elaborar un plan global para la producción y la distribución en la industria y en la agricultura tomando como base única el sistema de la propiedad de todo el pueblo" 23 . Seguidamente, Mao afirmaba que en una sociedad socialista, es decir, en etapa de transición al comunismo, la distribución según el trabajo, la producción mercantil, la ley del valor, esto es, los elementos del capitalismo, están conformes a las exigencias del desarrollo de las fuerzas productivas. Pero en la siguiente fase, estos principios y estas leyes no convendrán más con el desarrollo de las fuerzas productivas. Así, serán rotas por las fuerzas productivas y desaparecerán por sí mismas.

En el socialismo, según el Manual, no hay clases que entren en conflicto con los intereses comunistas. Por esto, el paso al comunismo no se realiza por medio de una revolución social. Por el contrario, para Mao si bien el paso al comunismo no significa el derrocamiento de una clase por otra clase, no se puede decir que no sea una revolución social, ya que "la substitución de una relación de producción por otra relación de producción es un salto cualitativo, es decir, una revolución" ${ }^{24}$. A su vez, la conversión del principio socialista "a cada uno según su trabajo" en el principio comunista "a cada uno según sus necesidades" constituye una revolución en el campo de las relaciones de producción.

\footnotetext{
22 Ídem. Pág. 46.

${ }^{23}$ Ídem. Pág. 51.

${ }^{24}$ Ídem. Pág. 64.
} 


\section{El Che y la crítica al Manual de Economía Política de la Unión Soviética}

Por último, Ernesto "Che" Guevara también aludió a este período que tercia entre el capitalismo y el comunismo en las críticas que le realizó al Manual de la Economía Política de la Unión Soviética ${ }^{25}$, en escritos que hace poco más de una década han sido publicados en formato de libro bajo el nombre de Apuntes críticos a la economía política ${ }^{26}$. En este libro, de la misma forma que en los textos de Mao anteriormente citados, se presentan diferentes párrafos de dicho Manual con los correspondientes comentarios que el Che hizo de cada uno.

Allí Guevara comenzaba citando la definición de período de transición del Manual, al igual que Mao, ("comienza con la instauración del poder proletario y termina cuando se da cima a la tarea de la revolución socialista, primera fase de la sociedad comunista."27), realizando asimismo una critica similar, ya que ésta hace desaparecer un tramo, por tanto Marx habla de transición del capitalismo al comunismo y el Manual sólo del capitalismo al socialismo. Guevara marcaba que lo que Marx denominó dictadura del proletariado es lo que se conoce ahora como socialismo, que sería una fase posterior al período de transición.

Por otro lado, Guevara afirmaba que en el período de transición las relaciones entre la clase obrera y el pequeño campesino son antagónicas, ya que este último "genera capitalismo a cada minuto" 28 . Aquí el Che estaba viendo la persistencia de la propiedad privada bajo el socialismo, y las contradicciones que ella conllevaba. Así, cuestionaba la interpretación de los revisionistas soviéticos que postulaban que las contradicciones entre la clase obrera y los campesinos trabajadores no son insuperables, y que los intereses de ambas clases coinciden, ya que ambas se sienten interesadas en suprimir la explotación y en que triunfe el socialismo. Para Guevara, como dijimos, las relaciones entre la clase obrera y el pequeño campesino son antagónicas, y son las relaciones económicas las marcan ese antagonismo. El poder proletario puede atemperarlas y convertirlas en no antagónicas, pero eso es un trabajo político.

En cuanto a las leyes económicas, en el Manual se planteaba que con la aparición y el desarrollo de la economía socialista, surge la ley económica fundamental del socialismo, esto es, el cambio radical que se opera en cuanto el fin de la producción; "en el sector socialista, la producción se lleva a cabo, no con el fin de obtener la ganancia capitalista, sino con el de elevar el bienestar material y el nivel cultural de los trabajadores"29. Al mismo tiempo, van creándose las condiciones necesarias para lograr este fin, mediante la aplicación de la industria y de una técnica avanzada. Pero Guevara revisó esta idea y postuló que en el socialismo esta ley fundamental es de orden moral, pero no puede ser nunca económica. Y afirmaba que, en el caso de existir, la ley económica fundamental del socialismo sería la planificación, que daría la posibilidad de interpretar y dirigir las leyes económicas de este período. Siguiendo al Manual, allí se planteaba que la propiedad social,

\footnotetext{
25 Toma las citas del Manual de la Economía Política. Editora Política. La Habana. 1963.

${ }^{26}$ Guevara, Ernesto: Apuntes críticos a la economía política. Ocean Sur. Bogotá. Colombia. 2007. Pág. 108.

27 Ídem. Pág. 108.

${ }^{28}$ Ídem. Pág. 111.

${ }^{29}$ Ídem. Pág. 113.
} 
que englobaba a todas las empresas del sector socialista, necesitaba que su desarrollo sea armónico. Así surgió la ley del desarrollo armónico (proporcional) de la economía nacional, la cual cumplía la función de regular el desarrollo de la producción socialista, donde el Estado establecía entre las ramas de la economía las proporciones necesarias para el triunfo del socialismo. Sin embargo, para el Che, esta ley era un aspecto de la planificación, y será muy distinta en un país con tendencia a la autarquía que en otro que aproveche la división internacional del trabajo, como Cuba o China. Sin embargo, en el sector socialista, afirma Guevara, había comenzado a actuar la ley de elevación constante de la productividad del trabajo, lo que consideraba una "aberración", por ser una ley del sistema capitalista.

En el socialismo, según los revisionistas soviéticos, debe cesar la ley del valor de la fuerza de trabajo, y así surgen nuevas relaciones de producción, es decir, relaciones de producción socialistas, donde actúa la ley económica de la distribución con arreglo al trabajo, según la cual cada trabajador recibe una remuneración correspondiente a su trabajo invertido. Pero Guevara también cuestionaba esto, planteándolo como muy vago e inexacto, ya que no es igual la inversión de trabajo de un ministro, de un maestro o de un obrero.

Mientras tanto -continuaba afirmándose en el Manual- la ley del valor actuaba en la economía nacional soviética pero únicamente como regulador de la producción en el sector de la pequeña economía mercantil y en el sector capitalista, no así en la producción socialista, es decir, en las empresas que se encuentran en manos del Estado, donde actuaba la ley de desarrollo planificado. Pero existían otras empresas socialistas aparte de las que a través de la nacionalización pasaron a ser de propiedad socialista estatal, y eran las cooperativas de las economías de los pequeños productores privados que también conformaban empresas socialistas pero de manera voluntaria. No obstante, estas cooperativas aún conservaban aspectos capitalistas, por ser de propiedad colectiva pero no social, como afirmaba Mao. Así, el Che remarcaba que la cooperativa engendra capitalismo, al desarrollar una estructura capitalista, y entra en contradicción con la sociedad.

Los revisionistas soviéticos reconocían la existencia de esta pequeña producción campesina de mercancías que origina la necesidad de recurrir al mercado y a la economía monetaria para la construcción del socialismo. El Che resaltaba que en el Manual dice "para" y no "durante"; "No se hace como palanca, (...) sino porque no hay más remedio o no se vio el remedio" 30 .

Así es como la política económica del período de transición del capitalismo al socialismo para los revisionistas tiene tres objetivos: primero, fortalecer las bases materiales de la alianza de clase entre los obreros y los campesinos. Segundo, interesar materialmente a los trabajadores de la ciudad y del campo en el incremento de la productividad de su trabajo. Y tercero, manejar en interés del socialismo palancas económicas tales como el comercio, el dinero, los precios, el crédito y el cálculo económico. Para Guevara, esto debía ser ampliamente discutido, especialmente lo último, relacionado con el uso de las categorías económicas del capitalismo ${ }^{31}$.

\footnotetext{
30 Ídem. Pág. 118.

${ }^{31}$ Ver Guevara, Ernesto: "El socialismo y el hombre en Cuba”. 1965.
} 
A raíz de este análisis, Guevara plateó, ya en 1965, una serie de críticas a la economía que llevaba adelante la Unión Soviética, lo que para muchos le costó su partida al Congo ese mismo año, conjunto con el alejamiento del gobierno cubano y la posterior emprendida hacia Bolivia en 1967, así como también que sus Apuntes críticos a la Economía Política permanecieron inéditos y ocultos aproximadamente por cuarenta años. En una carta enviada a Fidel Castro en 1965 y publicada en dicho libro, Guevara afirmaba: "Este primer período, -período de transición o período de la construcción de la sociedad socialista- los soviéticos y los checos pretenden haberlo superado; creo que objetivamente no es así, desde el momento en que todavía existen una serie de propiedades privadas en la Unión Soviética y, por supuesto, en Checoslovaquia"32.

\section{Palabras finales}

Volver sobre este tema, y hacerlo desde los clásicos del marxismo, nos permite no sólo pensar los diferentes problemas que se presentaron en las distintas experiencias socialistas del mundo, sino que además nos otorga herramientas factibles para comprender tanto la realidad de hoy como su dimensión histórica. En esto es esclarecedor el pensamiento del Che, que supo vislumbrar tanto los errores como los aciertos a los que se había arribado en la Unión Soviética y en otras experiencias socialistas como China y Cuba. Sus diferencias con la conducción de la Unión Soviética y con el propio gobierno cubano, su prematura muerte y el posterior encubrimiento de sus escritos hicieron que sus aportes no puedan ser utilizados con la importancia que hubieran merecido. Eso sólo hace aún más necesario estudiar y retomar su pensamiento.

\section{Bibliografía}

Arancibia, Simón: "Y límpiese bien la boca si dice Revolución". 27/09/2017. Tinta Roja. Disponible en: https://tinta-roja.com/2017/09/27/limpiese-bien-la-boca-dice-revolucion/.

Comité para el Centenario de la Revolución de Octubre: "Una obra inconclusa", en Lenin El Estado y la Revolución. Cuadernos de octubre. Edición Centenario. 2017.

Guevara, Ernesto: Apuntes críticos a la economía política. Ocean Sur. Bogotá. Colombia. 2007.

Lenin: El Estado y la Revolución. Editorial Agora. Buenos Aires. 2003.

Lenin: "La economía y la política de la dictadura del proletariado". Publicado en Pravda, núm. 250, 7 de noviembre de 1919.

Marx, Karl: Crítica del Programa de Gotha. Editorial Progreso. Moscú. 1977.

Mao, Zedong: La Construcción Del Socialismo. Editorial La Oveja Negra. Medellín. 1975.

Mao, Zedong: Discurso: Sobre problemas de filosofía. 18 de agosto de 1964.

Mao, Zedong: "Sobre la nueva democracia. Enero de 1940", en Obras Escogidas de Mao Tse-Tung. Tomo II. Editorial del Pueblo. Pekín. 1976.

\footnotetext{
${ }^{32}$ Fragmento de una carta que Guevara le enviara a Fidel Castro en abril de 1965, antes de su partida al Congo, incorporada a Modo de Prólogo en Guevara, Ernesto: Apuntes críticos a la economía política. Op. Cit. Págs. 9 y 10.
} 